EPJ Web of Conferences 58, 02010 (2013)

DOI: $10.1051 /$ epjconf/20135802010

(C) Owned by the authors, published by EDP Sciences, 2013

\title{
General Relativity by Kawaguchi geometry
}

\author{
Erico Tanaka ${ }^{1,2,3, a}$ \\ ${ }^{1}$ Department of Mathematics, Torino University, via Carlo Alberto 10, 10123 Torino, Italy \\ ${ }^{2}$ Department of Algebra and Geometry, Faculty of Science, Palacky University, 17. listopadu 12, 77146 \\ Olomouc, Czech Republic \\ ${ }^{3}$ Physics Department, Ochanomizu University, 2-1-1 Ootsuka Bunkyo, Tokyo, Japan
}

\begin{abstract}
We construct a parameterisation invariant Lagrange theory of fields up to second order by using multivector bundles and Kawaguchi geometry. In this setup, the spacetime is an dynamical object which is a submanifold of the greater manifold, and the actual spacetime is the solution of Euler-Lagrange equations. Such theory is a reasonable mathematical foundation to describe an extended theory of Einstein's general relativity, and is capable of being a stage for unification with other physical fields.
\end{abstract}

\section{Introduction}

The standard physical theory of fields is constructed on the fibre bundle structure, with the spacetime being the base manifold, and fields being its section. In such construction, the base manifold is chosen at the beginning, therefore becomes the "background", and naturally the target of study is mainly on the fields, not on its background spacetime. However, in such cases when one needs to consider spacetime itself as a dynamical object, it is apparent that this structure is insufficient. Such considerations arise especially in the past few decades research on the attempt to combine gravity with other fundamental forces or obtain its quantisation, where spacetime itself is the topic of discussion. In this letter, we will introduce a geometric foundation for considering such cases. The spacetime is introduced as a submanifold $\Sigma$ of a bigger manifold $M$ that is the total space of all dynamical variables to be considered, namely, physical fields and spacetime. This way of considering spacetime together with fields have been proposed in [1,2], for a local coordinate system using non-linear forms. Here we will use a structure called multivector bundles on manifolds. In contrast to the standard fibre bundle approach, we will assume no fibration, however, there still exists a natural bundle structure called the multivector bundle over $M$, which is an extension of the tangent bundle. To consider physically meaningful theory, we endow this multivector bundle a structure called Kawaguchi function $K$, and together with the total space $M$, it constructs a Kawaguchi manifold which is a natural extension of Finsler manifold. >From this Kawaguchi function, it is possible to construct a Kawaguchi differential form, which could be taken as a Lagrangian. This form integrated over a $k$-dimensional submanifold is the action of the whole system, and by the calculus of variation, we will obtain the Euler-Lagrange equations of the submanifold, which its extremal corresponds to the actual spacetime.

In the following, we will give the basic foundations, first for the case when the dimension of spacetime is 1 , namely the mechanics, and then for the fields.

\footnotetext{
a e-mail: eriko.tanaka@unito.it
} 


\section{First order mechanics}

In this section, we briefly review the Lagrange formulations of first order mechanics on Finsler manifold. We begin with the definition of a Finsler manifold.

Definition 2.1. Finsler manifold

Let $M$ be a $C^{\infty}$-differentiable manifold, $\left(T M, \tau_{M}, M\right)$ its tangent bundle, $T^{0} M:=T M \backslash 0$ the slit tangent bundle excluding the zero section from $T M$, and $(U, \varphi), \varphi=\left(x^{\mu}, y^{\mu}\right), \mu=1, \cdots, n$ a chart on $T M$. The $n$-dimensional Finsler manifold is a pair $(M, F)$ where $F$ is a $C^{0}$ function on $T M$ and $C^{\infty}$ function on $T^{0} M$, satisfying the following homogeneity conditions,

$$
F\left(x^{\mu}, \lambda y^{\mu}\right)=\lambda F\left(x^{\mu}, y^{\mu}\right), \quad \lambda>0 .
$$

This condition is also equivalent to the condition of Euler's homogeneous function theorem,

$$
\frac{\partial F}{\partial y^{\mu}} y^{\mu}=F
$$

Function with such properties is called a Finsler function.

We will use this minimal definition as our Finsler manifold.

Given a Finsler manifold, we obtain an important geometrical structure called a Hilbert form [3].

Definition 2.2. Hilbert form

The Hilbert form $\mathcal{F}$ is a 1 -form on $T^{0} M$, which in local coordinates are expressed by

$$
\mathcal{F}=\frac{\partial F}{\partial y^{\mu}} d x^{\mu}
$$

The Hilbert form is invariant with respect to the coordinate transformations by $x^{\mu} \rightarrow \tilde{x}^{\mu}=\tilde{x}^{\mu}\left(x^{\nu}\right)$, $y^{\mu} \rightarrow \tilde{y}^{\mu}=\frac{\partial \tilde{x}^{\mu}}{\partial x^{\nu}} y^{\nu}$, and acts as a Lagrangian of mechanics, when integrated over a one dimensional submanifold, a curve. Let $C$ be this parameterisable curve on $M$, and suppose we have an immersion $\sigma$ from an interval $I=\left[t_{i}, t_{f}\right] \in \mathbb{R}$ to this curve $C$, i.e., $C=\sigma(I)$. Then the Hilbert form defines a Finsler length $l_{C}^{\mathcal{F}}[\sigma]$ of $C$ by

$$
l_{C}^{\mathcal{F}}[\sigma]=\int_{\hat{\sigma}(I)} \mathcal{F}=\int_{t_{i}}^{t_{f}} \frac{\partial F}{\partial y^{\mu}}(\hat{\sigma}(t)) \frac{d\left(x^{\mu}(\sigma(t))\right.}{d t} d t=\int_{t_{i}}^{t_{f}} F(\hat{\sigma}(t)) d t
$$

where $\hat{\sigma}$ is a tangent lift of $\sigma$ defined by $\hat{\sigma}(t)=\left.\frac{d\left(x^{\mu} \circ \sigma\right)}{d t}\right|_{t}\left(\frac{\partial}{\partial x^{\mu}}\right)_{\sigma(t)}$. The Finsler length is invariant with respect to change of parameterisation $\rho=\sigma \circ \phi, \phi \in D i f f(\mathbb{R})$, which preserves orientation, and fixed at the boundary. We consider this as an action of mechanics.

The extremal of the Finsler length is the solution curve of the Euler-Lagrange equations. The Euler-Lagrange equation of (2.4) is obtained by considering a flow $\alpha_{s}, s \in \mathbb{R}$ on $M$, and comparing the value of (2.4) by every possible deformations. The variation of the action is then obtained by,

$$
\delta l_{C}^{\mathcal{F}}[\sigma]=\lim _{s \rightarrow 0} \frac{1}{s}\left[\int_{T \alpha_{s} \circ \widehat{\sigma} \circ i d_{I}^{-1}(I)} \mathcal{F}-\int_{\widehat{\sigma}(I)} \mathcal{F}\right]=\int_{\widehat{\sigma}(I)} L_{X} \mathcal{F} .=\int_{I} \hat{\sigma}^{*} L_{X} \mathcal{F} .
$$

$X$ is a vector field on $T M$ generated by $T \alpha_{s}, X=\frac{d\left(T \alpha_{s}\right)}{d s}$. In local coordinate expressions,

$$
X=\xi^{\mu} \circ \tau_{M}\left(\frac{\partial}{\partial x^{\mu}}\right)+\frac{\partial \xi^{\mu}}{\partial x^{v}} \circ \tau_{M} \cdot y^{v}\left(\frac{\partial}{\partial y^{\mu}}\right)
$$


where $\xi=\xi^{\mu} \frac{\partial}{\partial x^{\mu}}$ is a vector field generated by $\alpha_{s}, \xi=\frac{d \alpha_{s}}{d s}$. The extremal condition is,

$$
\hat{\sigma}^{*}\left\{\frac{\partial^{2} F}{\partial x^{\mu} \partial y^{\rho}} d x^{\rho}-d\left(\frac{\partial F}{\partial y^{\mu}}\right)\right\}=0, \quad \hat{\sigma}^{*}\left\{\left(\frac{\partial^{2} F}{\partial y^{\mu} \partial y^{\rho}}\right) d x^{\rho}\right\}=0 .
$$

The second equation becomes an identity by the homogeneity condition, and the first becomes the Euler-Lagrange equations, also by the homogeneity condition.

\section{First order fields}

Kawaguchi considered two directions of extending the Finsler geometry [4, 5]. The first in higher order derivatives and the second in the way to extend the parameter space, namely the spacetime. The latter is also called as areal metric geometry. For a higher order field theory, we need the combination.

We will first define the geometric structure on the total space of a $k$-multivector bundle $\left(\Lambda^{k} T M, \Lambda^{k} \tau_{M}, M\right)$. We will call this structure a first order $k$-areal Kawaguchi function.

Definition 3.1. Kawaguchi manifold (First order $k$-dimensional parameter space)

Let $M$ be a $n$-dimensional $C^{\infty}$-differentiable manifold, $(U, \varphi), \varphi=\left(x^{\mu}, y^{\mu_{1} \cdots \mu_{k}}\right), \mu, \mu_{1}, \cdots, \mu_{k}=1, \cdots, n$ be a chart on $\Lambda^{k} T M$, and $K \in C^{\infty}\left(\Lambda^{k} T M\right)$ with $k \leqslant n$ that satisfies the following homogeneity condition,

$$
K\left(x^{\mu}, \lambda y^{\mu_{1} \cdots \mu_{k}}\right)=\lambda K\left(x^{\mu}, y^{\mu_{1} \cdots \mu_{k}}\right), \quad \lambda>0 .
$$

We will call the function with such properties, a first order k-areal Kawaguchi function, and the pair $(M, K)$ a $n$-dimensional $k$-areal Kawaguchi manifold, or simply a Kawaguchi manifold.

The condition (3.1) is equivalent to the following,

$$
\frac{1}{k !} \frac{\partial K}{\partial y^{\mu_{1} \cdots \mu_{k}}} y^{\mu_{1} \cdots \mu_{k}}=K
$$

We will call the manifold $M$ a total space, in the sense it contains both spacetime and the field. Given a $n$-dimensional $k$-areal Kawaguchi manifold $(M, K)$, we can obtain a structure which we will call a Kawaguchi $k$-form. Kawaguchi $k$-form is constructed in accord with the condition (3.2), and gives the Lagrangian of a field theory when pulled back to the parameter space, namely the spacetime, by a certain parameterisation.

Definition 3.2. Kawaguchi $k$-form (first order field theory)

The Kawaguchi $k$-form $\mathcal{K}$ is a $k$-form on $\Lambda^{k} T M$, which in local coordinates are expressed by

$$
\mathcal{K}=\frac{1}{k !} \frac{\partial K}{\partial y^{\mu_{1} \cdots \mu_{k}}} d x^{\mu_{1}} \wedge \cdots \wedge d x^{\mu_{k}} .
$$

With this $k$-form,we can define a parameterisation invariant $k$-area of the submanifold $\Sigma$ of $M$.

Definition 3.3. Kawaguchi $k$-area

Consider the parameterisation $\sigma: P \rightarrow \Sigma \subset M$ where $P$ is a closed rectangle $P=\left[t_{i}^{1}, t_{f}^{1}\right] \times\left[t_{i}^{2}, t_{f}^{2}\right] \times$ $\cdots \times\left[t_{i}^{k}, t_{f}^{k}\right] \subset \mathbb{R}^{k}$. A Kawaguchi $k$-area of $\Sigma$ is defined by,

$$
\begin{aligned}
l_{\Sigma}{ }^{\mathcal{K}}[\sigma] & =\int_{\hat{\sigma}(P)} \mathcal{K}=\int_{t_{i}^{1}}^{t_{f}^{1}} \cdots \int_{t_{i}^{k}}^{t_{f}^{k}} \frac{1}{k !} \frac{\partial K}{\partial y^{\mu_{1} \cdots \mu_{k}}}(\hat{\sigma}(t)) y^{\mu_{1} \cdots \mu_{k}}(\hat{\sigma}(t)) d t^{1} \wedge \cdots \wedge d t^{k} \\
& =\int_{t_{i}^{1}}^{t_{f}^{1}} d t^{1} \cdots \int_{t_{i}^{k}}^{t_{f}^{k}} d t^{k} K\left(x^{\mu}(\sigma(t)), \frac{\partial\left(x^{\left[\mu_{1}\right.}(\sigma(t))\right)}{\partial t^{1}} \cdots \frac{\partial\left(x^{\left.\mu_{k}\right]}(\sigma(t))\right)}{\partial t^{k}}\right)
\end{aligned}
$$


where $\hat{\sigma}$ is a multi-tangent lift of $\sigma$ defined by

$$
\hat{\sigma}(t)=\left.\left.\frac{\partial\left(x^{\mu_{1}} \circ \sigma\right)}{\partial t^{1}}\right|_{t} \cdots \frac{\partial\left(x^{\mu_{k}} \circ \sigma\right)}{\partial t^{k}}\right|_{t}\left(\frac{\partial}{\partial x^{\mu_{1}}} \wedge \cdots \wedge \frac{\partial}{\partial x^{\mu_{k}}}\right)_{\sigma(t)}, \quad t \in P .
$$

Then we will have the following lemma,

Lemma 3.4. The area $l_{\Sigma}{ }^{\mathcal{K}}[\sigma]$ defined by (3.4) is invariant with respect to reparameterisation $\rho=\sigma \circ \phi$, $\phi \in \operatorname{Diff}\left(\mathbb{R}^{k}\right)$, where $\rho$ preserves orientation, and the boundary of $P$ is fixed.

The Euler-Lagrange expressions could be obtained by similar considerations.

Theorem 3.5. Variational formula of Kawaguchi $k$-area

The extremal conditions of (3.4) is given by,

$$
\hat{\sigma} L_{\hat{X}} \mathcal{K}=0
$$

where $\hat{X}$ is a vector field generated by the multi-tangent flow, $\Lambda^{k} T \alpha_{s}$, induced by a flow $\alpha_{s}$ on $M$.

In local coordinate expression, (3.6) becomes:

$$
\left\{\begin{array}{l}
\hat{\sigma}^{*}\left\{\left(\frac{\partial^{2} K}{\partial x^{\mu} \partial y^{\rho_{1} \cdots \rho_{k}}} d x^{\rho_{1}}-k d\left(\frac{\partial K}{\partial y^{\rho_{1} \cdots \rho_{k}}}\right)\right) \wedge d x^{\rho_{2} \cdots \rho_{k}}\right\}=0, \\
\hat{\sigma}^{*}\left\{y^{v \mu_{2} \cdots \mu_{k}}\left(\frac{\partial^{2} K}{\partial y^{\mu_{1} \cdots \mu_{k}} \partial y^{\rho_{1} \cdots \rho_{k}}}\right) d x^{\rho_{1} \cdots \rho_{k}}\right\}=0 .
\end{array}\right.
$$

The second equation becomes an identity by the homogeneity condition, and the first becomes the Euler-Lagrange equations, also by the homogeneity condition.

\section{Second order fields}

Here we will briefly describe the geometrical structures for higher order field theory. We will consider this by referring to the second order mechanics. The structure used for second order mechanics is the second order tangent bundle $\left(T^{2} M, \pi, M\right)$, which is not a vector bundle. It is constructed in the following way. Let $\left(T T M, \tau_{T M}, T M\right)$ be an iterated tangent bundle. Then, $\left(T^{2} M,\left.\tau_{T M}\right|_{T^{2} M}, T M\right)$ is a sub-bundle of $\left(T T M, \tau_{T M}, T M\right)$, defined by $T^{2} M=\left\{\forall v \in T T M \mid T \tau_{M}(v)=\tau_{T M}(v)\right\}$, where $\tau_{M}$ is a tangent projection $\tau_{M}: T M \rightarrow M$, and $\tau_{T M}$ is a tangent projection $\tau_{T M}: T T M \rightarrow T M$. Then the composed projection $\pi=\left.\tau_{M} \circ \tau_{T M}\right|_{T^{2} M}$ gives the second order tangent bundle. We similarly consider the second order multivector space by this construction.

Definition 4.1. The second order $k$-multivector bundle The structure $\left(\left(\Lambda^{k} T\right)^{2} M, \pi, M\right)$ with the following properties is called the second order $k$-multivector bundle.

$$
\begin{aligned}
& \text { 1. }\left(\Lambda^{k} T\right)^{2} M=\left\{\forall v \in \Lambda^{k} T \Lambda^{k} T M \mid \Lambda^{k} T \Lambda^{k} \tau_{M}(v)=\Lambda^{k} \tau_{\Lambda^{k} T M}(v)\right\} \\
& \text { 2. } \pi=\left.\Lambda^{k} \tau_{M} \circ \Lambda^{k} \tau_{\Lambda^{k} T M}\right|_{\left(\Lambda^{k} T\right)^{2} M} \text { is a surjective submersion. }
\end{aligned}
$$

The second order $k$-areal Kawaguchi manifold is defined as follows.

Definition 4.2. Second order $k$-areal Kawaguchi manifold

Let $(M, K)$ be a pair of $n$-dimensional $C^{\infty}$-differentiable manifold $M$ and a function $K \in$ $C^{\infty}\left(\left(\Lambda^{k} T\right)^{2} M\right), k \leqslant n$, which for a chart $(U, \varphi), \varphi=\left(x^{\mu}, y^{\mu_{1} \cdots \mu_{k}}, z^{I_{1} ; v_{2} \cdots v_{k}}, z^{I_{1} I_{2} ; v_{3} \cdots v_{k}}, \cdots, z^{I_{1} I_{2} \cdots I_{k}}\right)$, 
$\mu, \mu_{1}, \cdots, \mu_{k}, v_{2}, \cdots, v_{k}=1, \cdots, n$, and multi index notation: $I_{j}:=\mu_{j}^{i_{1}} \cdots \mu_{j}^{i_{k}}$, on $\left(\Lambda^{k} T\right)^{2} M$, satisfies the following second order homogeneity condition,

$$
\begin{aligned}
& K\left(x^{\mu}, \lambda y^{\mu_{1} \cdots \mu_{k}},(\lambda)^{2} z^{I_{1} v_{2}, \cdots v_{k}}+\lambda^{\nu_{2} \cdots v_{k}} y^{I_{1}},(\lambda)^{2} z^{I_{1} I_{2} v_{3}, \cdots v_{k}}+\lambda^{v_{3} \cdots v_{k}} y^{I_{1}} y^{I_{2}}, \cdots,(\lambda)^{2} z^{I_{1} I_{2} \cdots I_{k}}+\lambda^{0} y^{I_{1}} y^{I_{2}} \cdots y^{I_{k}}\right) \\
& =\lambda K\left(x^{\mu}, y^{\mu_{1} \cdots \mu_{k}}, z^{I_{1} v_{2}, \cdots v_{k}}, z^{I_{1} I_{2} v_{2}, \cdots v_{k}}, \cdots, z^{I_{1} I_{2} \cdots I_{k}}\right),
\end{aligned}
$$

for $\lambda>0$, and $\lambda^{v_{2} \cdots v_{k}}, \lambda^{v_{3} \cdots v_{k}}, \cdots, \lambda^{v_{k}}, \lambda^{0}$ being arbitrary constants. We will call the function with such properties, a second order $k$-areal Kawaguchi function, and the pair $(M, K)$ a $n$-dimensional second order k-areal Kawaguchi manifold.

Using the homogeneity condition as a guide, we can construct a geometric structure.

Definition 4.3. The second order Kawaguchi $k$-form $\mathcal{K}$ is a $k$-form on $\left(\Lambda^{k} T\right)^{2} M$, which in local coordinates are expressed by

$$
\begin{aligned}
& \mathcal{K}=\frac{1}{k !} \frac{\partial K}{\partial y^{\mu_{1} \cdots \mu_{k}}} d x^{\mu_{1} \cdots \mu_{k}}+\frac{2}{(k-1) !} \frac{\partial K}{\partial z^{I_{1} v_{2} \cdots v_{k}}} d y^{I_{1}} \wedge d x^{\nu_{2} \cdots v_{k}}+\frac{2}{(k-2) !} \frac{\partial K}{\partial z^{I_{1} I_{2} v_{3} \cdots v_{k}}} d y^{I_{1}} \wedge d y^{I_{2}} \wedge d x^{\nu_{3} \cdots v_{k}} \\
& +\cdots+2 \frac{\partial K}{\partial z^{I_{1} I_{2} \cdots I_{k}}} d y^{I_{1}} \wedge \cdots \wedge d y^{I_{k}} .
\end{aligned}
$$

We used the abbreviation such as $d x^{\mu_{1} \cdots \mu_{k}}:=d x^{\mu_{1}} \wedge \cdots \wedge d x^{\mu_{k}}, d y^{I_{1}} \wedge d x^{\nu_{2} \cdots \nu_{k}}:=d y^{I_{1}} \wedge d x^{\nu_{2}} \wedge \cdots \wedge d x^{\nu_{k}}$. Now we can define the $k$-area;

Definition 4.4. The second order Kawaguchi k-area is defined by,

$$
l_{\Sigma} \mathcal{K}[\sigma]=\int_{\sigma^{2}(P)} \mathcal{K}=\int_{t_{i}^{1}}^{t_{f}^{1}} d t^{1} \int_{t_{i}^{2}}^{t_{f}^{2}} d t^{2} \cdots \int_{t_{i}^{k}}^{t_{f}^{k}} d t^{k} K\left(\sigma^{2}(t)\right), \quad t \in P,
$$

where $\sigma^{2}$ is a second order lift of parameterisation $\sigma \rightarrow \Sigma$, defined by the iterated tangent lift with condition $\Lambda^{k} T \Lambda^{k} \tau_{M}(\sigma(t))=\Lambda^{k} \tau_{\Lambda^{k} T M}(\sigma(t)), t \in P$.

Lemma 4.5. The area $l_{\Sigma}{ }^{\mathcal{K}}[\sigma]$ defined by (4.5) is invariant with respect to reparameterisation $\rho=\sigma \circ \phi$, $\phi \in \operatorname{Diff}\left(\mathbb{R}^{k}\right)$, where $\rho$ preserves orientation, and the boundary of $P$ is fixed.

This is the action of second order field theory. To obtain the Euler-Lagrange equations, we consider a flow $\alpha_{s}$ on $M$, and it induces a flow $\left(\Lambda^{k} T\right)^{2} \alpha_{s}$ on $\left(\Lambda^{k} T\right)^{2} M$. Then we will have the following:

Theorem 4.6. Variational formula of second order Kawaguchi $k$-area

The extremal conditions of (4.5) are given by,

$$
\sigma^{2} L_{X^{2}} \mathcal{K}=0
$$

where $X^{2}$ is a vector field generated by the flow, $\left(\Lambda^{k} T\right)^{2} \alpha_{s}$.

To find the solution of Euler-Lagrange equations is equivalent to find the extremal of the action, which is a submanifold that corresponds to our spacetime embedded into a greater space together with other dynamical variables. General relativity could be regarded as an embedded theory, when a semi-Riemannian metric is induced on this submanifold.

\section{References}

[1] T. Ootsuka, arXiv:1206.6040v1 (2012)

[2] R. Yahagi, T. Ootsuka, E. Tanaka, Soryuushiron Kenkyu 13 (2012)

[3] W.H.C. S. S. Chern, K.S. Lam, Lectures on Differential Geometry (World Scientific, Singapore, 2000)

[4] A. Kawaguchi, Periodica Mathematica Hungarica 7, 291 (1976)

[5] A. Kawaguchi, Fac. Sci. Hokkaido (1964) 
